\title{
A test for the too-big-to-fail hypothesis for European banks during the financial crisis
}

\author{
Abstract \\ Motivated by the theoretical prediction of the opportunistic behaviour of large banks that \\ face expected public intervention, we test a full and a partial form of the too-big-to-fail (TBTF) \\ hypothesis. The full form of the hypothesis implies the increase in the risk undertakings and \\ profitability of banks that exceed a certain dimension; the partial form of the hypothesis \\ implies only an augmented risk appetite of large banks compared to their smaller \\ counterparts. The examined area is the European banking industry, whose behaviour is \\ observed over the first wave of the present financial crisis (2007-2009). The estimation of a \\ quadratic fit that links change in a bank's credit risk profile and profitability retention rates \\ with a bank's size suggests the existence of a partial form of the TBTF hypothesis. However, a \\ more precise, local rolling-window estimation of the size sensitivities reveals that large banks \\ whose liabilities exceed approximately $2 \%$ of the country of origin's GDP ( $15 \%$ of our sample), \\ and an increase in credit risk profile show, vis-à-vis their smaller counterparts, a superior \\ capability of retaining higher ROA scores. With the caveats of our investigation, we interpret \\ these results as evidence of a full form of the TBTF hypothesis.
}

